\title{
First bite syndrome as presenting symptom of parapharyngeal adenoid cystic carcinoma
}

\author{
A DEGANELLO ${ }^{1}$, G MECCARIELLO $^{1}$, M BUSONI $^{1}$, A FRANCHI $^{2}$, O GALLO $^{1}$ \\ ${ }^{1}$ Otolaryngology / Head and Neck Surgery Clinic, Department of Surgical Sciences, and ${ }^{2}$ Section of Human \\ Pathology, Department of Critical Care and Surgery, University of Florence, Italy
}

\begin{abstract}
Introduction: First bite syndrome refers to the development of pain in the parotid region after the first bite of each meal.

Case report: A man was referred to our institution with first bite syndrome as his only symptom. Magnetic resonance imaging of the head and neck revealed a deep lobe parotid mass in close contact with the external carotid artery. Computed tomography guided fine needle biopsy indicated adenoid cystic carcinoma. Total parotidectomy was performed, with en bloc resection of the infiltrated external carotid artery and a selective neck dissection of levels Ib to III. High-weight ion radiotherapy was administered post-operatively.

Conclusion: First bite syndrome has hitherto only been described following parapharyngeal space surgery. We present the first case of parotid gland adenoid cystic carcinoma presenting with first bite syndrome as the only symptom. The presence of first bite syndrome should prompt the clinician to investigate the parapharyngeal space and deep lobe of the parotid gland, especially in the absence of other signs or symptoms.
\end{abstract}

Key words: Carcinoma; Adenoid Cystic; Parotid Gland; Signs And Symptoms; Facial Pain

\section{Introduction}

In 1955, Gardner and Abdullah ${ }^{1}$ were the first to describe parotid pain following superior cervical ganglionectomy. In 1986, Haubrich named this symptom first bite syndrome. ${ }^{2}$

First bite syndrome has previously been described only as a complication of parapharyngeal space surgery. $^{3-6}$ The main symptom is pain in the parotid and jaw, often with radiation to the ear, which lasts a few seconds and occurs with the first bite of each meal. ${ }^{3}$ The symptoms are always worse with the first bite, and improve and resolve with subsequent bites, only to recur at the start of the next meal. Some patients experience pain when salivating or thinking about eating. The pain is also described as being at its worst with the first meal of the day. ${ }^{4}$ These symptoms often start five to seven days after parapharyngeal surgery, and in many cases persist despite medical and surgical intervention.

In this study, we present a case of first bite syndrome occurring as a unique and isolated presenting symptom of adenoid cystic carcinoma of the deep lobe of the parotid gland in an adult male, and we discuss the possible pathophysiology.

\section{Case report}

A 32-year-old man was referred to our institution with a 12-month history of pain in the right parotid region at the beginning of each meal. His medical history was negative for chronic diseases, previous surgery and tumours.

Clinical inspection and palpation demonstrated no signs of facial nerve dysfunction, no palpable masses, and no mucosal lesions within the head or neck.

The patient's symptom fitted the clinical picture of first bite syndrome.

Ultrasound evaluation of the neck and major salivary glands was performed, revealing a solid mass with irregular edges within the deep lobe of the right parotid gland.

Contrast-enhanced magnetic resonance imaging of the neck showed a multilobulated mass in the deep lobe of the right parotid gland, with involvement of the intraglandular external carotid artery (Figure 1). A computed tomography guided fine needle aspiration biopsy was suggestive of adenoid cystic carcinoma.

A total extended parotidectomy was performed under general anaesthesia, with en bloc resection of 
(a)

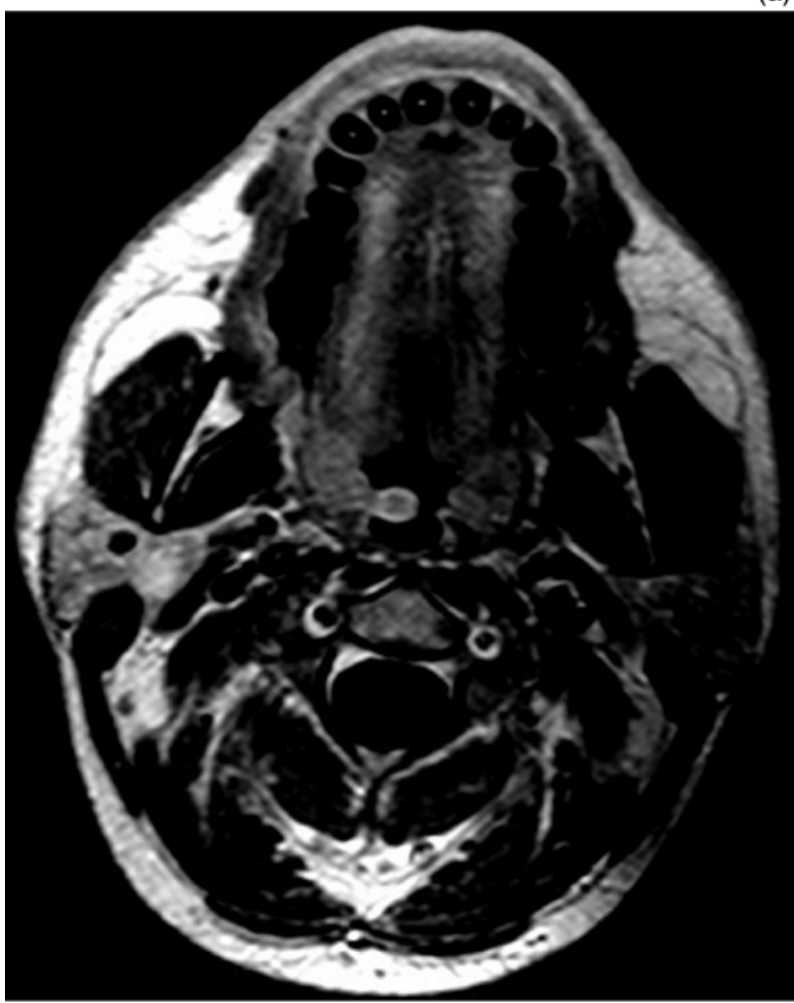

(b)

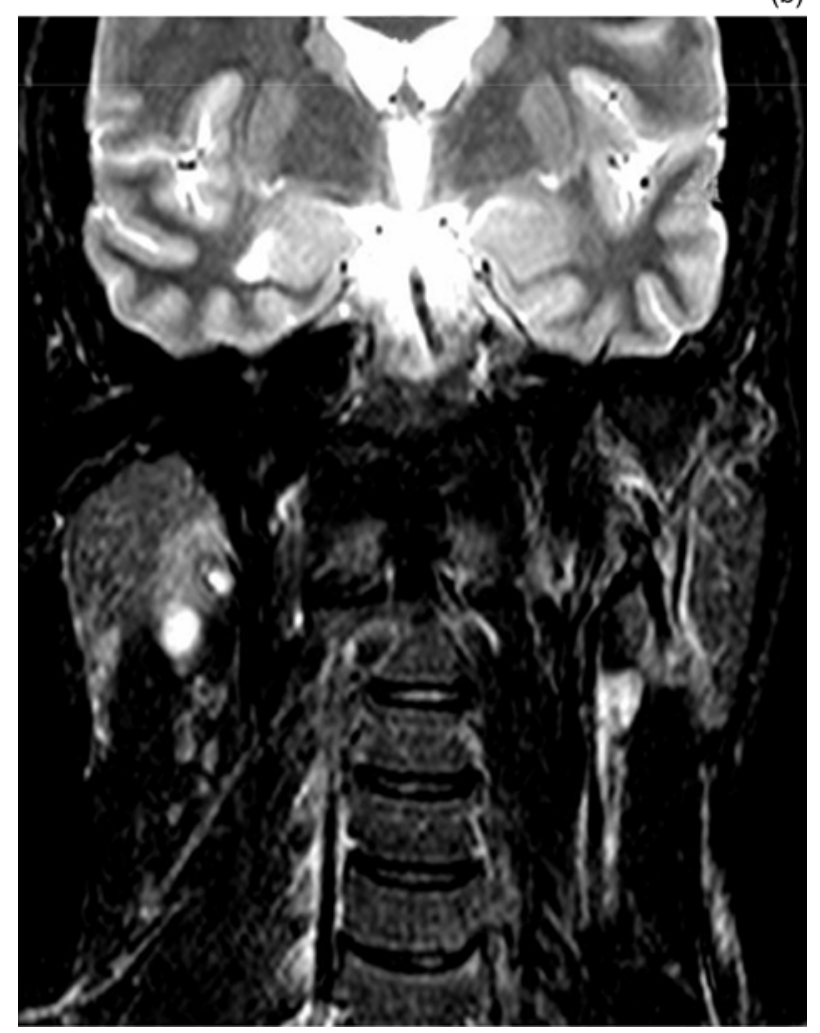

FIG. 1

(a) Axial and (b) coronal magnetic resonance imaging scans showing a multilobulated tumour mass in the deep lobe of the right parotid gland.

the infiltrated external carotid artery and the posterior belly of the digastric muscle, together with neck dissection of levels Ib to III.
Macroscopically, the tumour was firmly adherent to the under-surface of the common trunk of the facial nerve. Therefore, the mass was carefully dissected under microscopic view, preserving the nerve. Vascular clips were then placed in the field to assist post-operative extra boost targeting. Frozen section analysis of the tissue peeled off the nerve confirmed tumour perineural spread.

Post-operatively, no signs of facial nerve impairment were documented. Surprisingly, the patient did not experience further first bite syndrome, despite having undergone parapharyngeal surgery.

Histopathological examination revealed adenoid cystic carcinoma with a predominant cribriform pattern, less than 5 per cent of which was composed of solid areas. The tumour infiltrated the adventitia of the external carotid artery and diffusely involved the nerve sheaths of medium-sized and small nerves (Figure 2). No metastases were found in 30 resected lymph nodes.

The patient completed adjuvant fast neutron radiotherapy. The total dose was $15.3 \mathrm{~Gy}$, fractioned in 1.67 Gy doses and given three times a week. No signs of tumour recurrence were found after 38 months of follow up, and the patient remained painfree.

\section{Discussion}

First bite syndrome usually presents as a complication of parapharyngeal space surgery. Its pathophysiology is not completely clear.

Netterville et $a l^{4}{ }^{4}$ described a series of 46 vagal paragangliomas and reported post-operative first bite syndrome in nine patients, each of whom had a transection of the sympathetic chain and/or loss of sympathetic function (as manifested by Horner's syndrome). Kawashima et $a l .{ }^{6}$ reviewed 29 patients with parapharyngeal space tumour, nine of whom developed first bite syndrome; eight of these patients had undergone resection of the cervical sympathetic chain and/or external carotid artery.

- First bite syndrome has been described only as a complication of parapharyngeal surgery

- The presented case shows that first bite syndrome can develop without previous parapharyngeal surgery

- In the presented case, first bite syndrome appeared as a presenting symptom and resolved after parapharyngeal surgery; thus, the pathophysiological mechanisms of this syndrome may vary from previous descriptions

- Clinicians should investigate the parotid gland and parapharyngeal space in patients presenting with pain matching first bite syndrome 
(a)
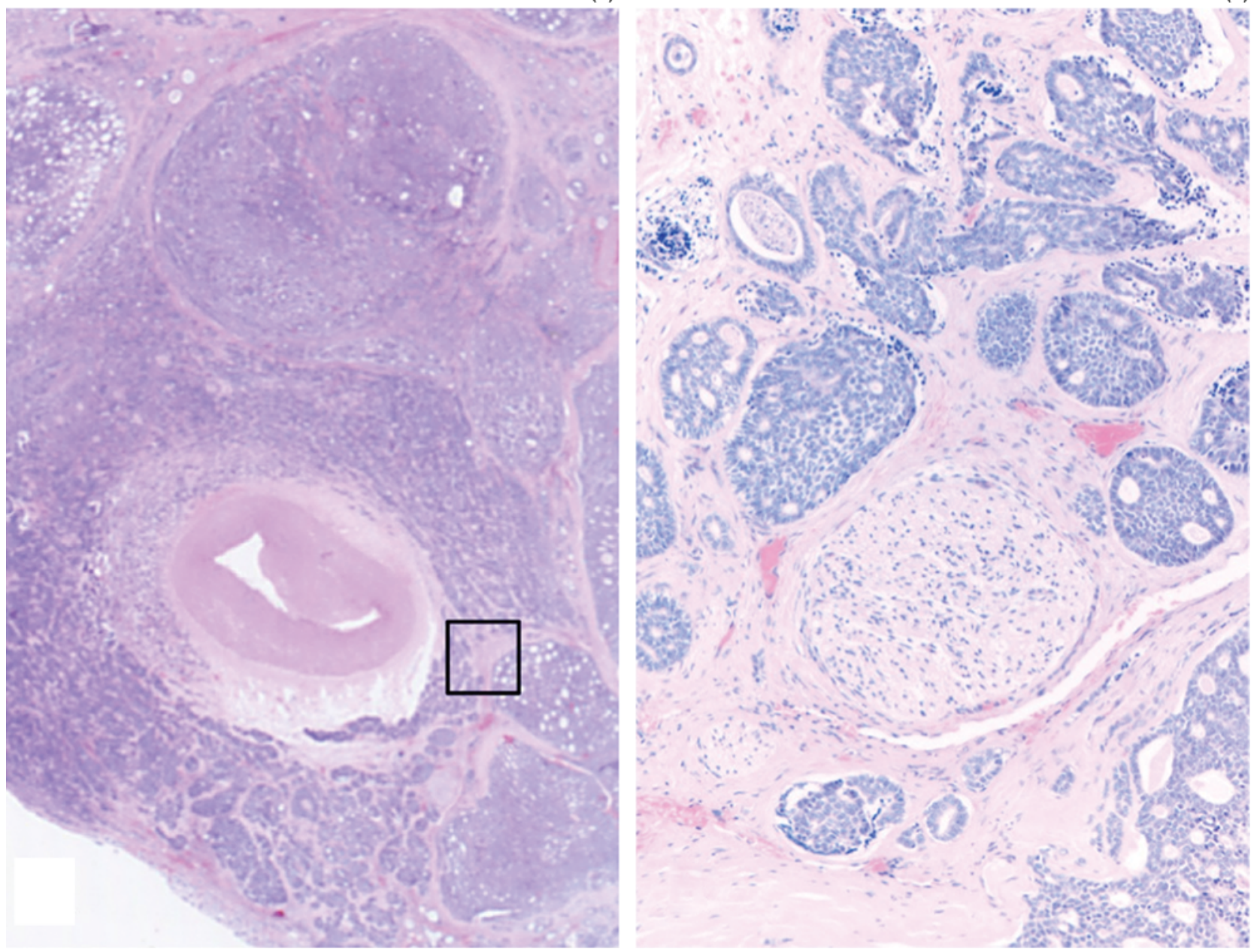

FIG. 2

(a) Photomicrograph showing the external carotid artery infiltrated by tumour $(\mathrm{H} \& \mathrm{E} ; \times 2,5)$. (b) Enlarged section from part (a) showing the sympathetic carotid plexus infiltrated by tumour $(\mathrm{H} \& \mathrm{E} ; \times 10)$.

These findings strongly suggest that loss of parotid sympathetic innervation causes first bite syndrome. Damage or removal of cervical sympathetic innervation results in a denervation supersensitivity of the sympathetic receptors that control myoepithelial cells within the gland. A supramaximal response by myoepithelial cells is elicited on cross-stimulation by the release of parasympathetic neurotransmitters, induced by chewing and biting (Figure $3 b$ ).

In our patient, the onset of first bite syndrome without Horner's syndrome may be explained by lack of tumour involvement of the sympathetic chain within the parapharyngeal space, and by denervation supersensitivity of the sympathetic network accompanying the external carotid artery. However, if this was the case, why then would first bite syndrome disappear after parapharyngeal surgery with resection of the external carotid artery? Was it because the entire parotid gland had been removed, ${ }^{3}$ or because first bite syndrome was sustained by some other mechanism?

In our patient, we cannot exclude an 'irritative' sympathetic myoepithelial hyper-response due to the presence of an adenoid cystic carcinoma involving the external carotid artery. However, if this mechanism was sufficient in itself to cause first bite syndrome, why has this syndrome never been reported as a presenting symptom of other parapharyngeal tumours involving the external carotid artery; indeed, why is it not relatively common in cases of parotid adenoid cystic carcinoma? The explanation may be that irritation of the sympathetic system is not sufficient to hyper-simulate myoepithelial cells (Figure 3c).

We must consider that myoepithelial cells are innervated by both autonomic nervous systems, ${ }^{7,8}$ and that published evidence shows that the sympathetic nervous system does not antagonise the parasympathetic with respect to salivary output. ${ }^{89}$ Furthermore, parasympathetic receptors can be cross-stimulated by sympathetic neurotransmitters; in an animal model in which the submaxillary gland had been denervated by transection of the chorda tympani, external adrenaline stimulation caused salivary release. ${ }^{9}$

Our case may supply evidence that tumour irritation of the sympathetic system, together with concomitant tumour denervation of the parasympathetic system, 


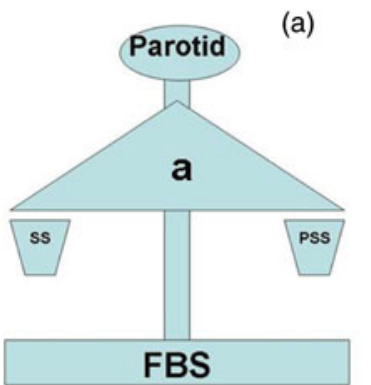

(c)

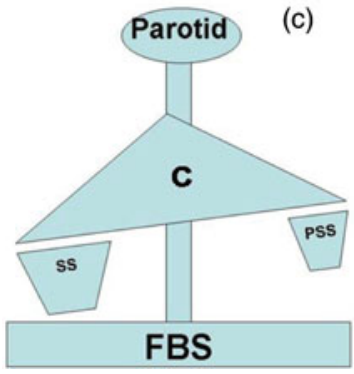

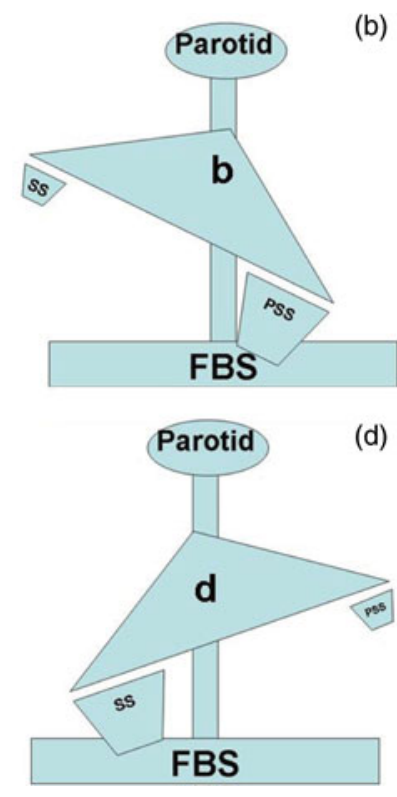

FIG. 3

Diagrams showing balance of sympathetic and parasympathetic parotid innervation in first bite syndrome. (a) Normal myoepithelial cell activation by both sympathetic and parasympathetic systems; no first bite syndrome. (b) Denervation of the sympathetic system causing first bite syndrome by parasympathetic cross-stimulation. (c) Irritative stimulation of the sympathetic system without parasympathetic denervation; no first bite syndrome. (d) Irritative stimulation of the sympathetic system and concomitant denervation of the parasympathetic system, causing first bite syndrome by sympathetic cross-stimulation.

can cause myoepithelial supersensitivity and hyperresponsiveness, resulting in first bite syndrome as a presenting symptom (Figure 3d).

Finally, we cannot exclude the possibility that first bite syndrome occurring unrelated to parapharyngeal surgery may be overlooked in general clinical practice, either because it is masked by other symptoms or because it is classified as simply 'pain'. To investigate this possibility, we reviewed our institutional experience. From 1980 to 2008, we treated 47 cases of adenoid cystic carcinoma of the parotid gland, eight of which were located in the deep lobe, and none of which had referred pain with characteristics matching first bite syndrome. During the same period, we treated 25 parapharyngeal space tumours, five of which were malignant, and none of which had first bite syndrome as a presenting symptom; seven of these patients developed first bite syndrome postoperatively.

Here, we present the first reported case of parotid gland adenoid cystic carcinoma with first bite syndrome as the only presenting symptom. The presence of pain fitting the pattern of first bite syndrome should not be overlooked. If suspicion of first bite syndrome arises, with no other symptoms and signs, we recommend scrupulous investigation of the parapharyngeal space and deep lobe of the parotid gland, in order to detect possible malignancy.

\section{References}

1 Gardner WJ, Abdullah AF. Parotid pain following superior cervical ganglionectomy: a clinical example of the antagonistic action of the parasympathetic and sympathetic systems. Am J Med Sci 1955;230:65-9

2 Haubrich WS. The first-bite syndrome. Henry Ford Hosp Med J 1986;34:275-8

3 Chiu AG, Cohen JI, Burningham AR, Andersen PE, Davidson BJ. First bite syndrome: a complication of surgery involving the parapharyngeal space. Head Neck 2002;24:996-9

4 Netterville JL, Jackson CG, Miller FR, Wanamaker JR, Glasscock ME. Vagal paraganglioma: a review of 46 patients treated during a 20-year period. Arch Otolaryngol Head Neck Surg 1998;124:1133-40

5 Chijiwa H, Mihoki T, Shin B, Sakamoto K, Umeno H, Nakashima T. Clinical study of parapharyngeal space tumours. J Laryngol Otol 2009;31:100-3

6 Kawashima Y, Sumi T, Sugimoto T, Kishimoto S. First bite syndrome: a review of 29 patients with parapharyngeal space tumor. Auris Nasis Larynx 2008;35:109-13

7 Emmelin N, Muren A, Stromblad R. Effect of anastomosis between the hypoglossal and chorda-lingual nerves on the supersensitivity of the denervated submaxillary gland. Acta Physiol Scan 1957; $\mathbf{4 1 : 1 8 - 3 4}$

8 Kontis TC, Johns ME. Anatomy and physiology of the salivary glands. In: Bailey BJ, ed. Head and Neck SurgeryOtolaryngology, 2nd edn. Philadelphia: Lippincott-Raven, 1998;531-9

9 Lung MA. Autonomic nervous control of myoepithelial cells and secretion in submandibular gland of anaesthetized dogs. J Physiol 2003;546:837-50

Address for correspondence:

Dr Alberto Deganello,

Otolaryngology / Head and Neck Surgery Clinic,

Department of Surgical Sciences,

University of Florence,

V le Morgagni 85 Florence, Italy

Fax: + 39055435649

E-mail: adeganello@hotmail.com

Dr A Deganello takes responsibility for the integrity of the content of the paper

Competing interests: None declared 